Supporting Information

\title{
Fabrication of a Novel Nanofiltration Membrane with Enhanced Performance via Interfacial Polymerization through the Incorporation of a New Zwitterionic Diamine Monomer
}

Shao-Lu Li, Xinyao Shan, Yuanfei Zhao, and Yunxia $\mathrm{Hu}^{*}$

State Key Laboratory of Separation Membranes and Membrane Processes, School of Materials Science and Engineering, National Center for International Research on Membrane Science and Technology, Tianjin Polytechnic University, Tianjin 300387, P. R. China

*Corresponding author: Tel: +86-22-83955129, e-mail: yunxiahu@tjpu.edu.cn 


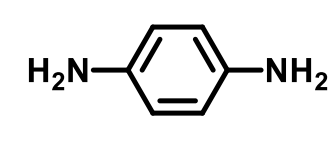

PPD

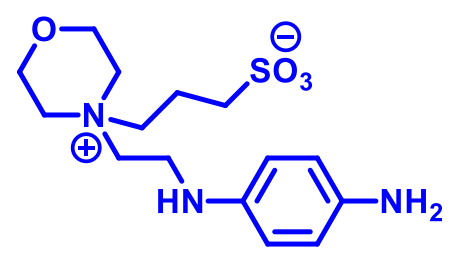

PPD-MEPS

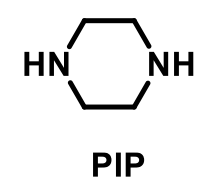

$\Theta$

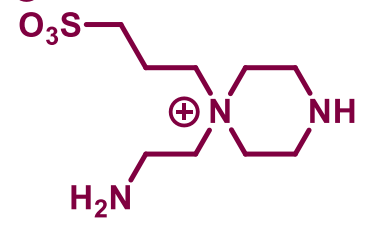

AEPPS

Scheme S1. The chemical structure of these four molecules.
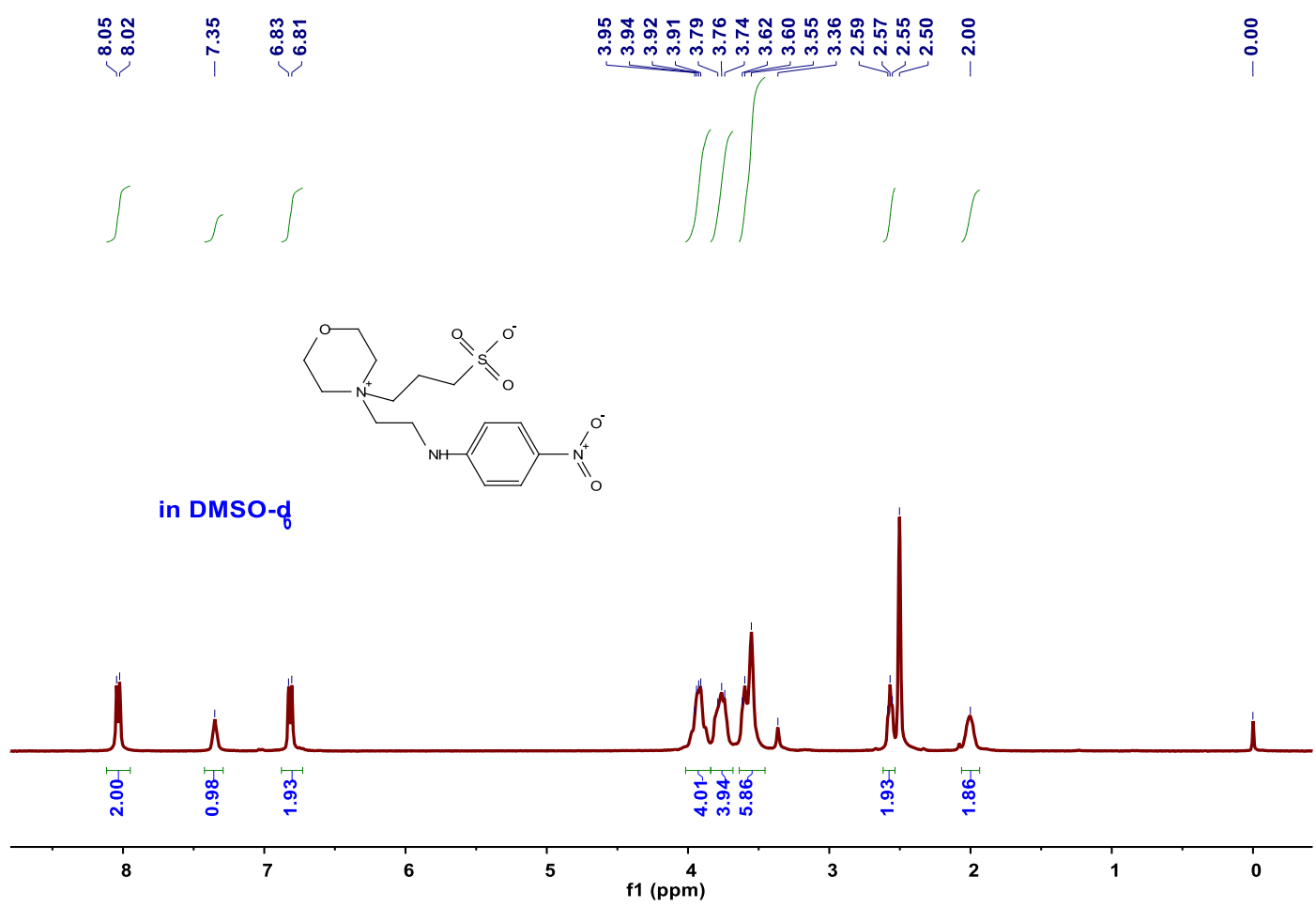

Figure S1. ${ }^{1} \mathrm{H}$ NMR spectrum of 3-(4-(2-((4-nitrophenyl)amino)ethyl)morpholino-4ium)propane-1-sulfonate in DMSO- $\mathrm{d}_{6}$. 


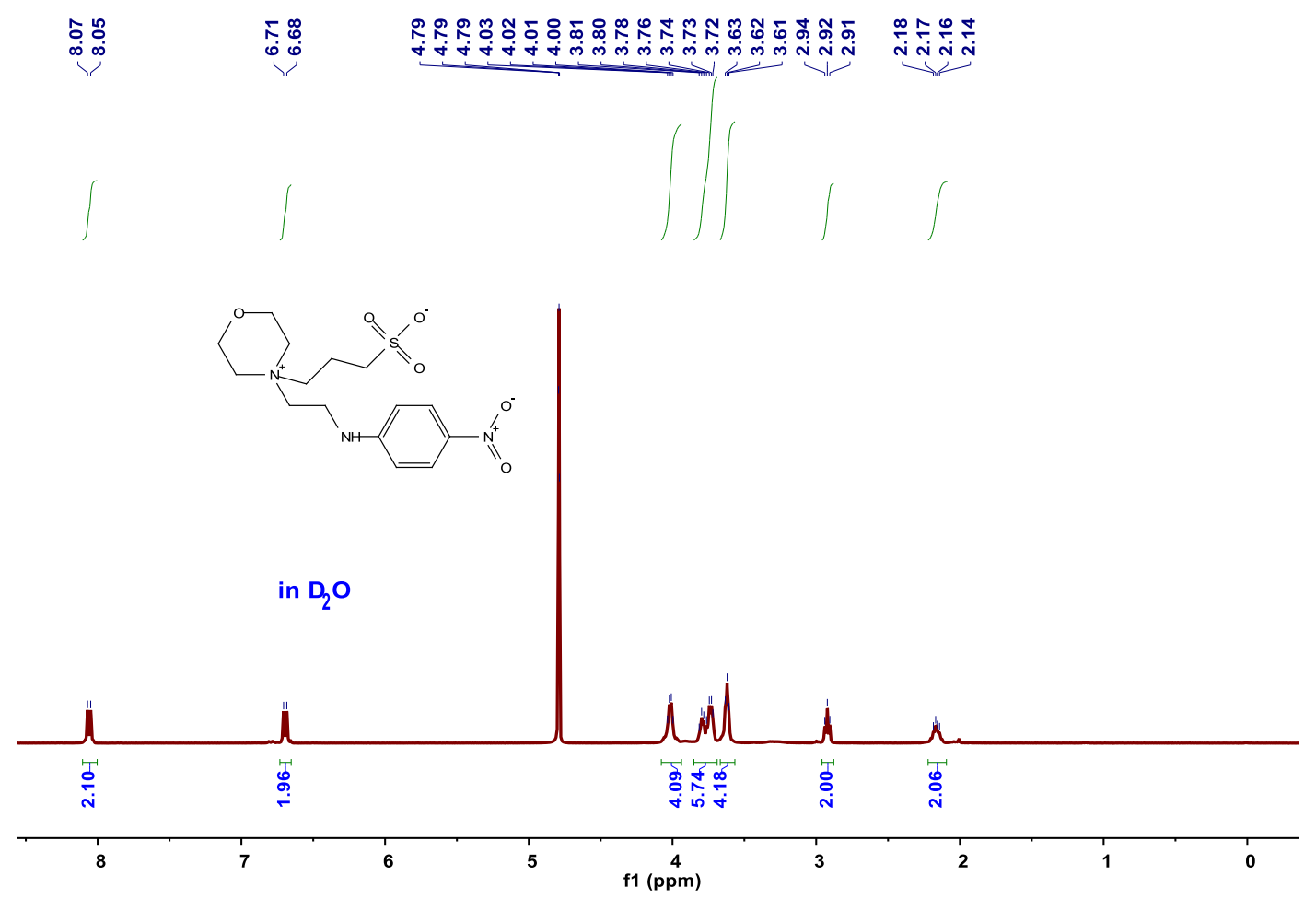

Figure S2. ${ }^{1} \mathrm{H}$ NMR spectrum of 3-(4-(2-((4-nitrophenyl)amino)ethyl)morpholino-4ium)propane-1-sulfonate in $\mathrm{D}_{2} \mathrm{O}$. 
$M=373$

LSL-PS-PND 51 (0.876) Cm (49:65)

100

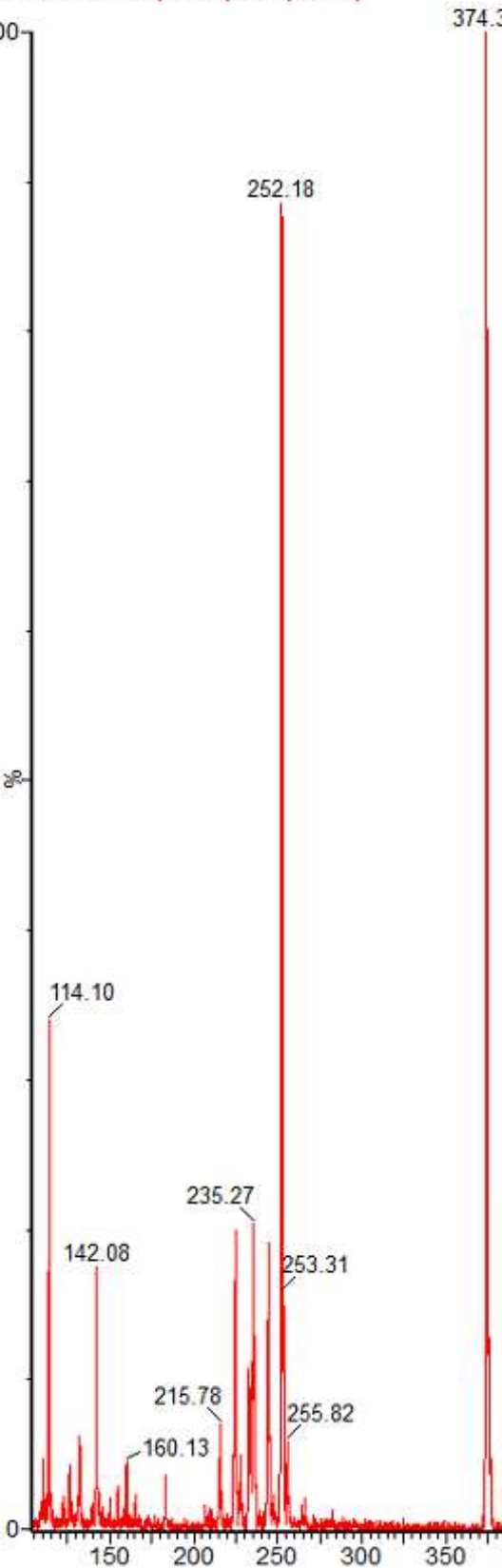

Figure S3. ESI-MS spectrum of 3-(4-(2-((4-nitrophenyl)amino)ethyl)morpholino-4ium)propane-1-sulfonate.
ACQ-SQD2\#LCA429

1: Scan ES+

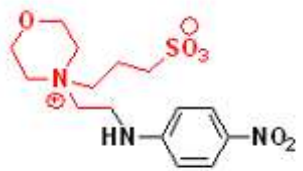

Exact Mass: 373.

393.35

579.78

747.32
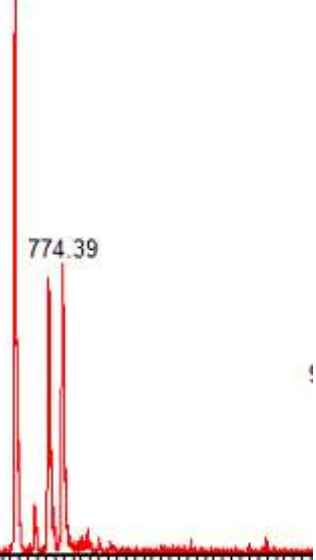

00 
$M=343$

LSL-PS-PPD 37 (0.633)

$100{ }^{114.18}$

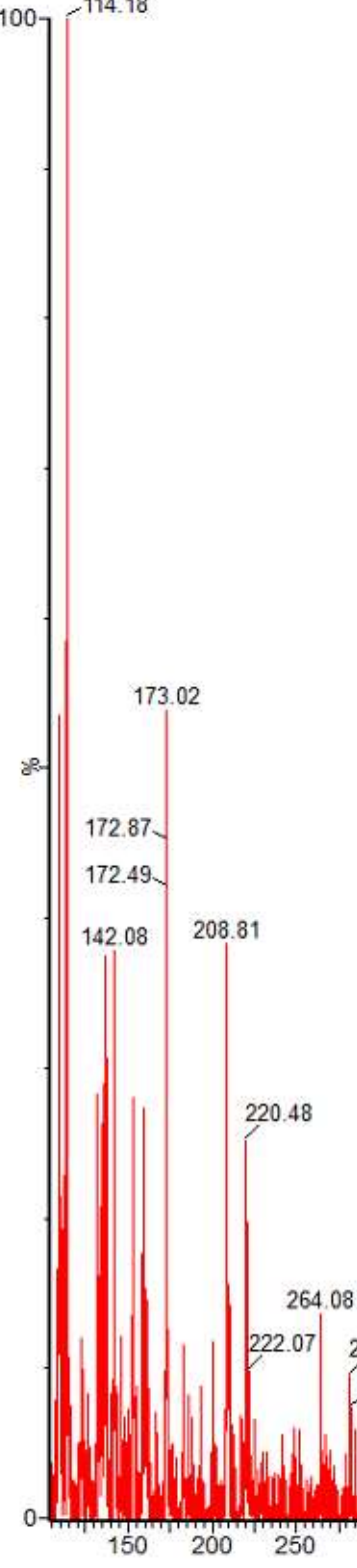

ACQ-SQD2\#LCA429

344.37

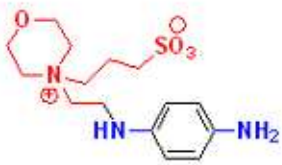

Exact Mass: 343.2

345.28

360.29

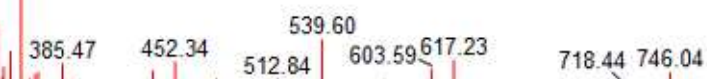

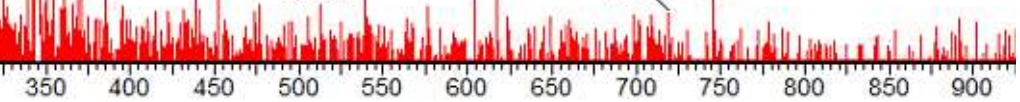

Figure S4. ESI-MS spectrum of the PPD-MEPS. 


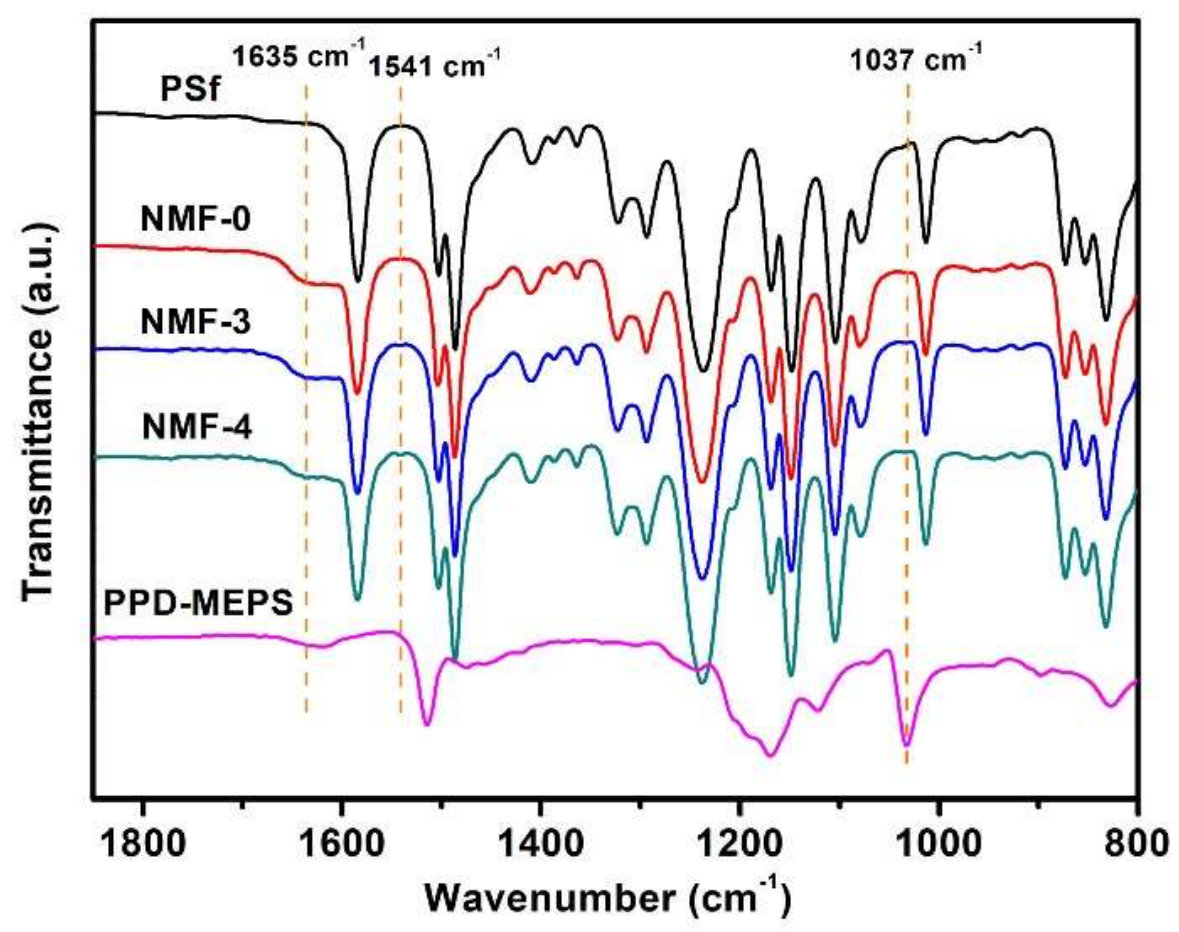

Figure S5. ATR-FTIR spectra of PSF-UF, NFM-0, NFM-3, NFM-4 and monomer PPDMEPS.

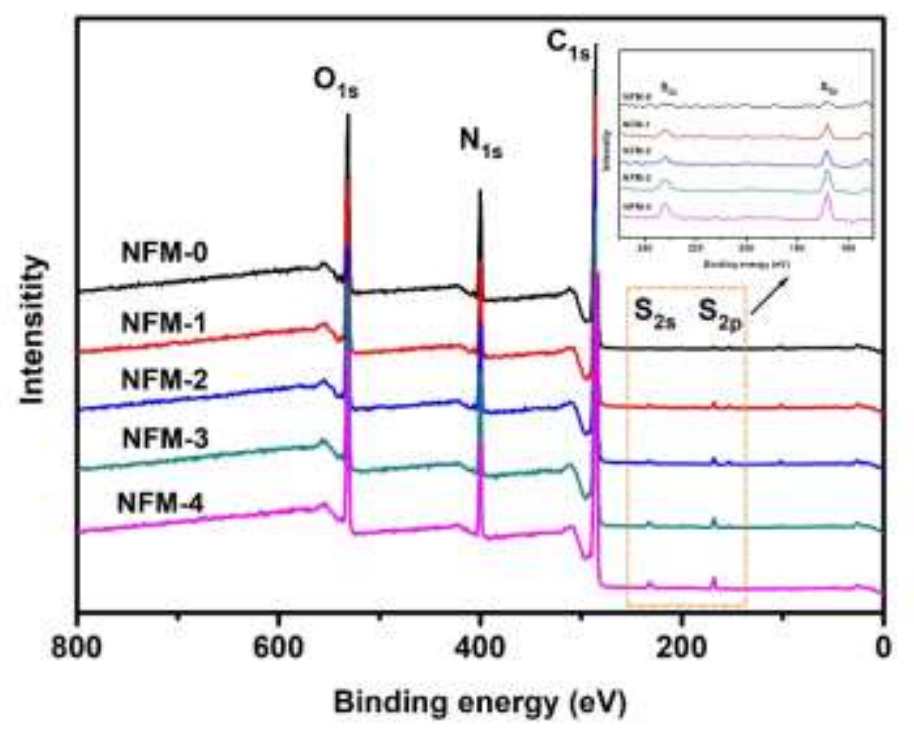

Figure S6. XPS survey spectrum of the TFC polyamide membranes including NFMs. 


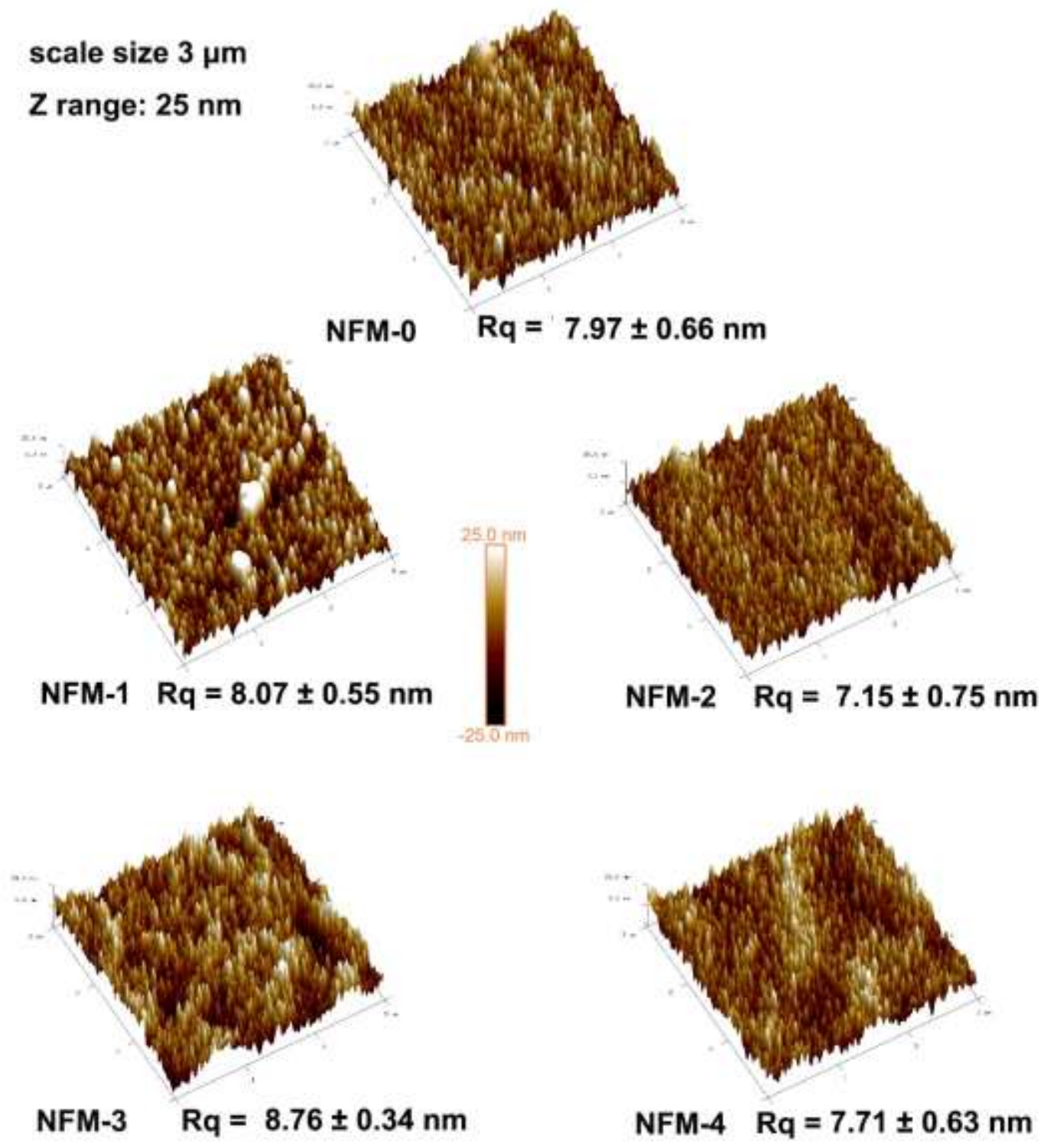

Figure S7. AFM images of the TFC NFMs. 
NFM-1
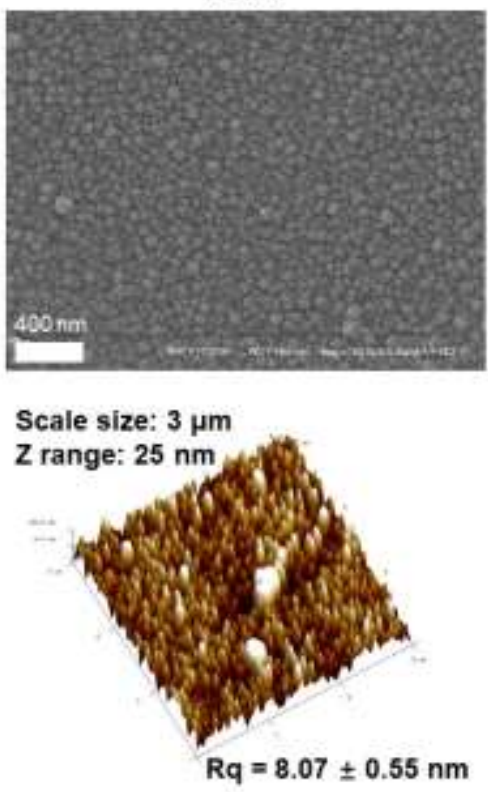

NFM-5
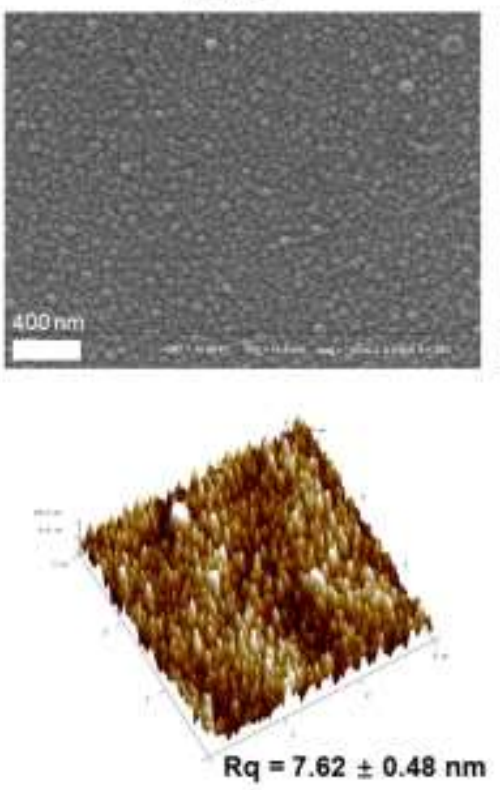

NFM-P
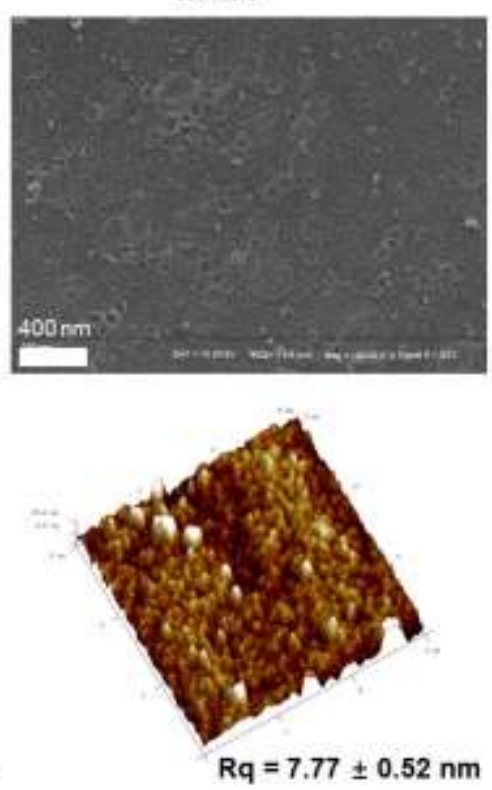

Figure S8. Surface morphologies of the fabricated polyamide NF membranes (membranes NFM-1, NFM-5, and NFM-P). NFM-5 was prepared from PIP (1.0 wt $\%$ ) and PPD-MEPS $(1.0 \%)$, while NFM-P is prepared from sole PPD-MEPS (1.0 wt \%).

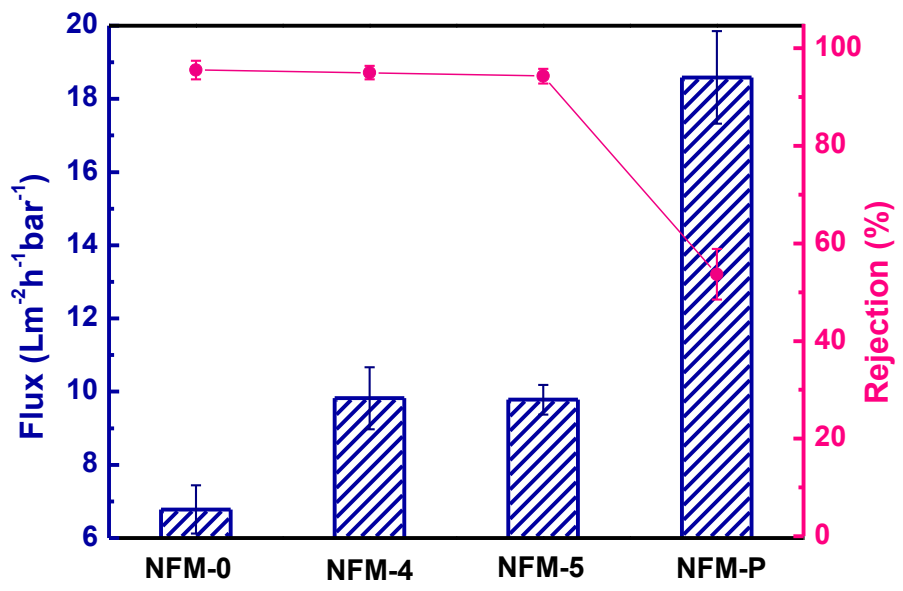

Figure S9. Nanofiltration performances including flux and rejection of NFMs, NFM-5 was prepared from PIP (1.0 wt \%) and PPD-MEPS (1.0\%), while NFM-P is prepared from sole PPD-MEPS (1.0 wt\%) following the similar procedure. 

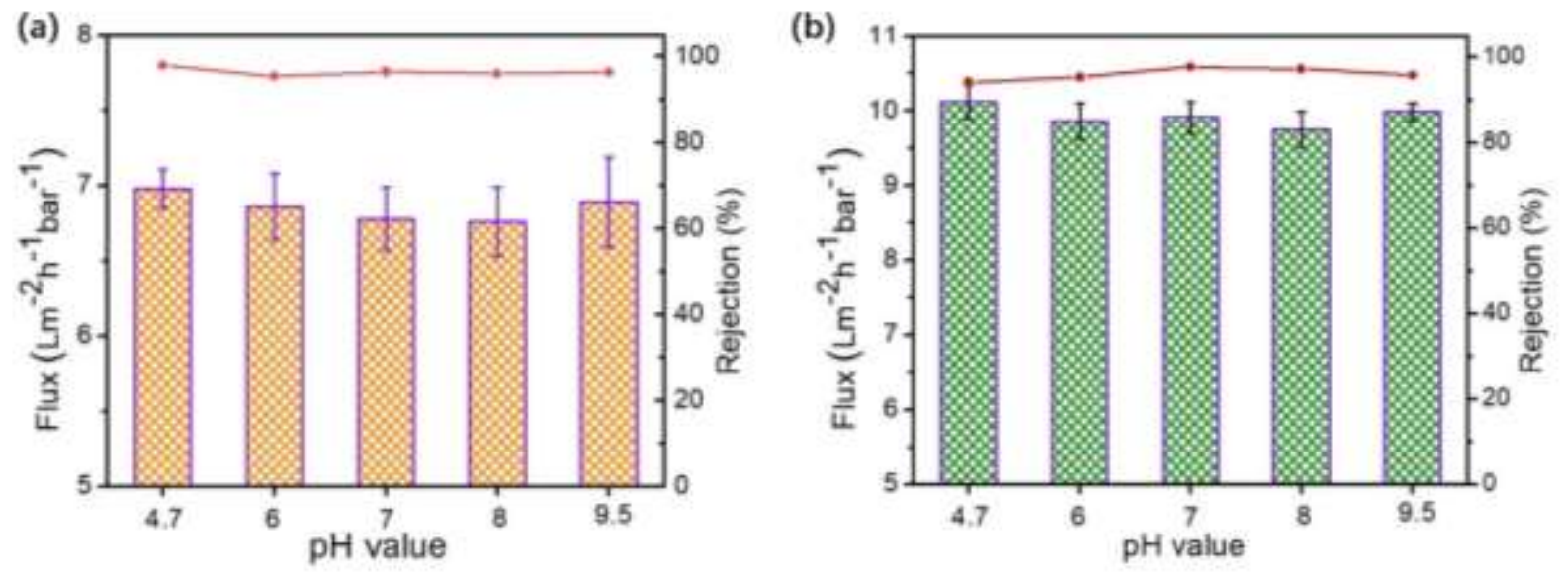

Figure S10. Water flux and $\mathrm{Na}_{2} \mathrm{SO}_{4}$ salt rejection of the fabricated nanofiltration membrane including a) NFM- 0 and b) NFM- 4 at $25^{\circ} \mathrm{C}$ under 5 bar when the feed solution had a $\mathrm{pH}$ in the range of 4.7 and 9.5 . 